Check for updates

Cite this: RSC Adv., 2019, 9, 2302

\title{
Scoparone protects neuronal cells from oxygen glucose deprivation/reoxygenation injury
}

\author{
Chunfang Wu, † Ting Li, $\uparrow$ Baihui Zhu, Ruiming Zhu, Youran Zhang, Fanglan Xing \\ and Yong Chen (iD)*
}

Ischemic stroke is one of the leading causes of death and disability in the world. The cerebral ischemia/ reperfusion (I/R) injury is considered as the major molecular mechanism in the pathogenesis of ischemic stroke. Scoparone, a major constituent of Artemisia capillaries, has been found to exhibit protective effects against I/R-induced myocardial injury. However, the role of scoparone in cerebral I/R injury has not been elucidated. In the current study, the hippocampal neurons were subjected to oxygen-glucose deprivation/reperfusion (OGD/R) to simulate I/R injury in vitro. The results showed that scoparone improved OGD/R-induced inhibitory effect on cell viability of hippocampal neurons. Scoparone displayed anti-oxidative activity as proved by the decreased levels of reactive oxygen species (ROS) and malondialdehyde (MDA), and increased activities of superoxide dismutase (SOD) and glutathione peroxidase (GPX) in OGD/R-induced hippocampal neurons. In addition, cell apoptosis was markedly decreased after scoparone treatment in OGD/R-induced hippocampal neurons. The expression of bax was significantly decreased, while bcl-2 expression was increased in the scoparone pretreated hippocampal neurons. Furthermore, the expressions of nuclear factor E2-related factor 2 (Nrf2) and heme oxygenase-1 (HO-1) were obviously induced by scoparone. Knockdown of Nrf2 by siRNA transfection dramatically attenuated the protective effects of scoparone on OGD/R-induced hippocampal neurons. Collectively, scoparone protected hippocampal neurons from OGD/R-induced injury via activating $\mathrm{Nrf2/HO}-1$ signaling pathway, suggesting that scoparone might be a potential agent for the ischemic stroke therapy.

Received 1st December 2018

Accepted 9th January 2019

DOI: $10.1039 / c 8 r a 09867 k$

rsc.li/rsc-advances mechanisms of ischemic stroke. ${ }^{5,6}$ Reperfusion after ischemia can recover blood flow and the supply of oxygen as well as many other energy materials. However, this process bears risks of worsening the original brain damage caused by ischemia. ${ }^{2}$ That is because that various free radicals are released during the reperfusion progress. ${ }^{2,7}$ The excessive free radicals may result in oxidative damage, leading to the activation of inflammatory cascade reaction, promotion of cellular apoptosis and breakdown of blood-brain barrier, which are much more sophisticated than ischemia alone. ${ }^{5,8}$ Therefore, intervention of $\mathrm{I} / \mathrm{R}$ injury is critical for treatment of patients with ischemic stroke.

Scoparone, a major constituent of Artemisia capillaries, has a variety of biological properties including anti-tumor, ${ }^{9}$ antiinflammatory, ${ }^{10}$ anti-oxidant, ${ }^{11}$ and neuro-protective activities. ${ }^{12}$ Previous study has demonstrated that scoparone exhibits protective effects against I/R-induced myocardial injury via the inhibition of oxidative stress and cell apoptosis. ${ }^{13}$ Another study showed that scoparone suppressed the lipopolysaccharidestimulated inflammatory responses in BV-2 microglial cells. ${ }^{14}$ However, the role of scoparone in cerebral $\mathrm{I} / \mathrm{R}$ injury remains unclear. Thus, we investigated the effect of scoparone on oxygenglucose deprivation/reperfusion (OGD/R)-induced neuron death and the underlying mechanism.
Department of Neurology, Huaihe Hospital of Henan University, East Gate Avenue 115\#, Kaifeng 475000, Henan province, China. E-mail: chenyongkf@163.com; Tel: +86-0371-23906882

$\dagger$ These authors contributed equally to this work. 


\section{Materials and methods}

\subsection{Cell culture}

Primary hippocampal neuronal cells were isolated from the brains of neonatal Sprague-Dawley rats as previously described..$^{15}$ The isolated hippocampal tissues were dissected and dissociated in $0.25 \%$ trypsin-EDTA at $37{ }^{\circ} \mathrm{C}$ for $10 \mathrm{~min}$. Afterward, the cells were suspended with Dulbecco's Modified Eagle's Medium (DMEM; Gibco Laboratories, Grand Island, NY, USA) and incubated for $4 \mathrm{~h}$. Then the medium was changed with neurobasal medium (Gibco) containing 2\% B27 supplement (Gibco) and 1\% glutamine (Gibco). Cells were maintained in a humidified $5 \% \mathrm{CO}_{2}$ atmosphere at $37^{\circ} \mathrm{C}$. Half of the medium was changed twice a week. The following experiments were performed on 8- to 10-d-old cultures. All animal procedures were performed in accordance with the Guidelines for Care and Use of Laboratory Animals of Huaihe Hospital of Henan University (Kaifeng, China) University and approved by the Animal Ethics Committee of Huaihe Hospital of Henan University.

\subsection{OGD/R model and treatment}

To initiate OGD, cells were moved to serum and glucose-free DMEM and maintained in an ischemic condition with $5 \%$ $\mathrm{CO}_{2}$ and $95 \% \mathrm{~N}_{2}$ for $3 \mathrm{~h}$ at $37^{\circ} \mathrm{C}$. After OGD exposure, the cells were cultured in normal medium under normoxic condition with $5 \% \mathrm{CO}_{2}$ and $95 \%$ air for $24 \mathrm{~h}$ for reoxygenation. Cells were pretreated with different concentrations of scoparone $(\geq 98 \%$; Sigma-Aldrich, St. Louis, MO, USA) for $2 \mathrm{~h}$, and then subjected to $\mathrm{OGD} / \mathrm{R}$. The chemical structure of scoparone was shown in Fig. 1A.

\subsection{Cell transfection}

The Nrf2 siRNA (si-Nrf2) and scramble siRNA (si-NC) were designed and synthesized by GenePharma (Shanghai, China). The siRNAs were transiently transfected into hippocampal neurons using Lipofectamine 2000 transfection reagent (Invitrogen, Carlsbad, CA, USA) according to the manufacturer's protocol.

\subsection{Cell viability assay}

Hippocampal neuronal cells were seeded into 96-well plates at 5 $\times 10^{3}$ cells per well and cultured under normal conditions for $24 \mathrm{~h}$. The cells were pretreated with or without scoparone (25, 50 , and $100 \mu \mathrm{M})$, followed by OGD/R stimulation or only treated with scoparone $(0,25,50,100,150$ and $200 \mu \mathrm{M})$ for $48 \mathrm{~h}$. Cell viability was evaluated using MTT assay. In brief, $20 \mu \mathrm{l}$ of MTT ( $5 \mathrm{mg} \mathrm{ml}{ }^{-1}$; Sigma) was added to each well at the end of the indicated treatments. After incubation at $37^{\circ} \mathrm{C}$ for another $4 \mathrm{~h}$, the culture medium was removed and $100 \mu \mathrm{l}$ of dimethyl sulfoxide (DMSO; Sigma) was added to each well to dissolve the formazan precipitate. Absorbance was measured at a wavelength of $450 \mathrm{~nm}$ with a microplate reader (Synergy H1 Hybrid Reader, Bio-Tek, Winooski, VT, USA).

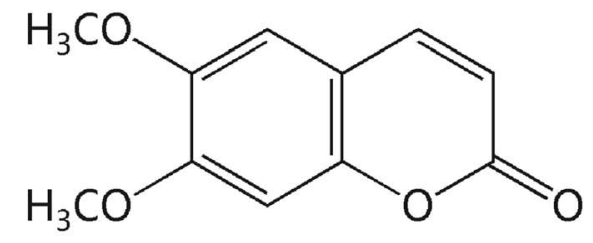

B
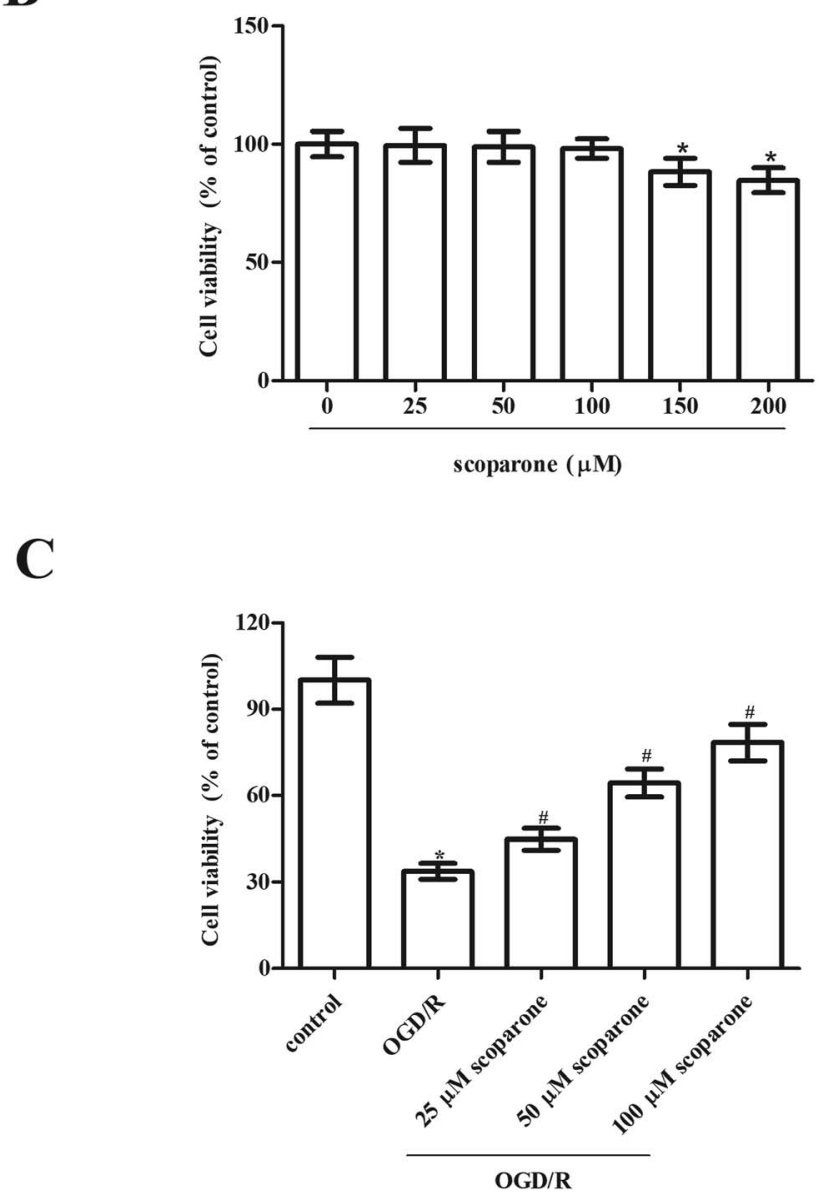

Fig. 1 Effect of different treatments on cell viability of hippocampal neurons. (A) The chemical structure of scoparone. (B) Cell viability of hippocampal neurons after treatment with different doses of scoparone $(0,25,50,100,150$ and $200 \mu \mathrm{M})$ for $48 \mathrm{~h}$. "*" indicates significant difference in comparison to the control group, $p<0.05$. (C) Cell viability of hippocampal neurons after $\mathrm{OGD} / \mathrm{R}$ induction with or without the pretreatment with scoparone $(25,50$ and $100 \mu \mathrm{M})$ for $2 \mathrm{~h} . \mathrm{n}$ $=3$. " $*$ " indicates significant difference in comparison to the control group, $p<0.05$. "\#" indicates significant difference in comparison to the $O G D / R$ induction group, $p<0.05$.

2.5 Measurement of reactive oxygen species (ROS), superoxide dismutase (SOD), malondialdehyde (MDA), and glutathione peroxidase (GPx)

After different treatments, hippocampal neurons were collected for biochemical analysis. The ROS generation, MDA level and activities of SOD and GPx were measured using commercial kits 

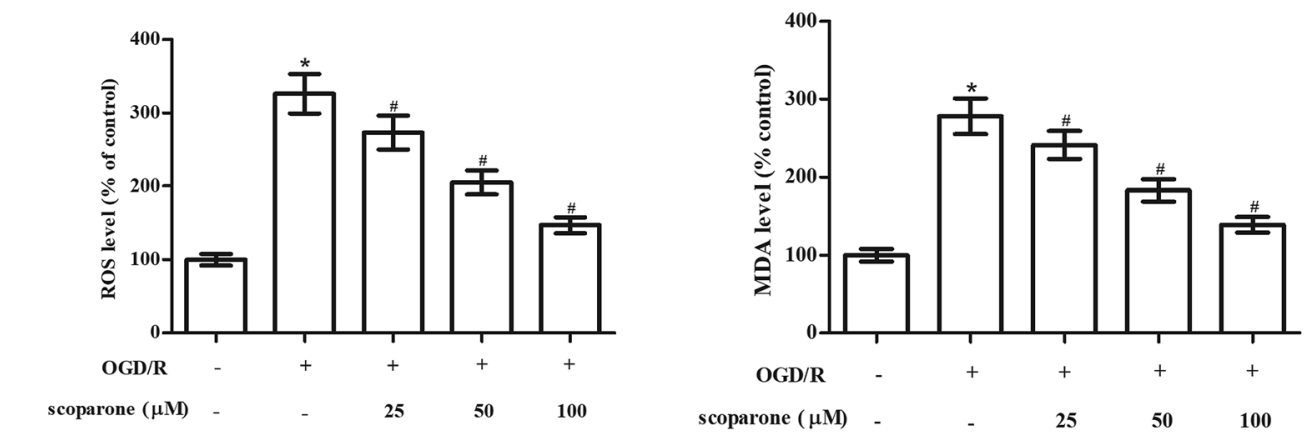
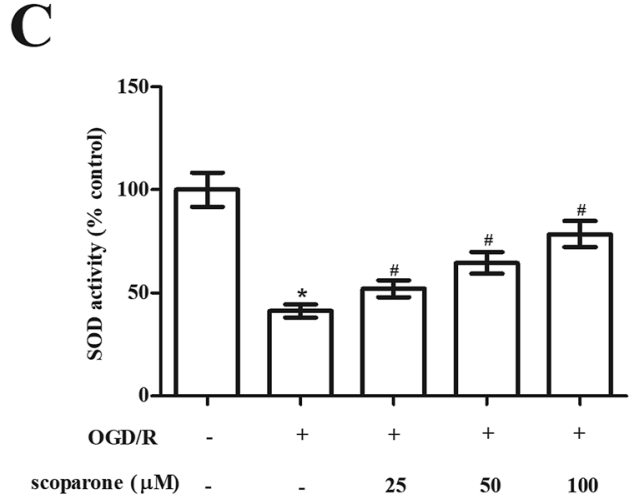

D

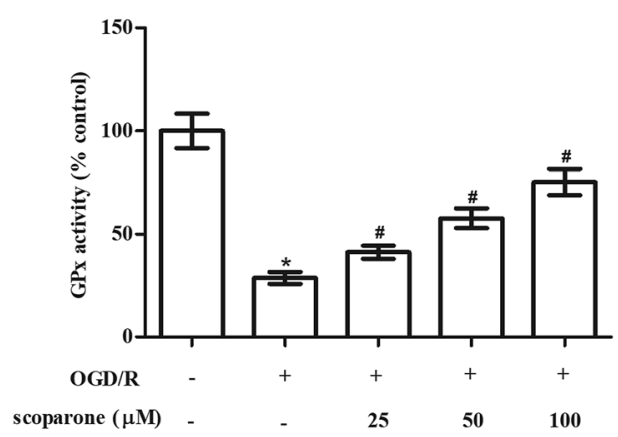

Fig. 2 Effect of different treatments on oxidative stress in hippocampal neurons. Hippocampal neurons subjected to OGD/R induction with or without the pretreatment with scoparone (25, 50 and $100 \mu \mathrm{M})$ for $2 \mathrm{~h}$. (A) Generation of ROS. (B) Content of MDA. (C) Activity of SOD. (D) Activity of GPx. $n=3$. "*" indicates significant difference in comparison to the control group, $p<0.05$. "\#" indicates significant difference in comparison to the $O G D / R$ induction group, $p<0.05$.

(Beyotime Biotechnology, Shanghai, China) according to the manufacturer's instruction.

\subsection{Cell apoptosis}

Cell apoptosis of hippocampal neurons was determined by cell death detection ELISA kit obtained from Roche (Palo Alto, CA, USA) according to the manufacturer's instructions.

\subsection{Western blot assay}

For the extraction of whole cell lysates, cells were homogenized with radioimmunoprecipitation assay (RIPA) lysis buffer (Thermo Scientific) supplemented with protease and phosphatase inhibitors (Abcam, Cambridge, MA, USA). Then a BCA protein assay kit (Thermo Scientific) was utilized to quantify the total protein concentrations. The protein samples $(30 \mu \mathrm{g})$ were denatured and separated by $10-12 \%$ SDS-PAGE. After that, proteins were transferred onto a polyvinylidene difluoride membrane (Invitrogen) at $4{ }^{\circ} \mathrm{C}$. Membranes were then blocked with $5 \%$ nonfat milk for $1 \mathrm{~h}$ at $37^{\circ} \mathrm{C}$ and then incubated overnight at $4{ }^{\circ} \mathrm{C}$ with the following antibodies: anti-bax $(1: 1000$ dilution, Santa Cruz Biotechnology, Santa Cruz, CA, USA), antibcl-2 (1: 1000 dilution, Santa Cruz), anti-nuclear factor E2related factor 2 (Nrf2) $(1: 1000$ dilution, Abcam, Cambridge, MA, USA), anti-heme oxygenase-1 (HO-1) $(1: 1000$ dilution, Abcam), and anti- $\beta$-actin (1:1000 dilution, CST). Subsequently, the membranes were incubated with horseradish peroxidase (HRP)-conjugated secondary antibodies (1: 5000 dilution, CST) at room temperature for $1 \mathrm{~h}$. An enhanced chemiluminescence (ECL) detection system (Thermo Scientific) was used to visualize the protein bands.

\subsection{Statistical analysis}

The results were expressed as means \pm SEM of at least three independent replications. The SPSS version 18.0 software (SPSS Inc., Chicago, IL, USA) was used for statistical analysis. The oneway analysis of variance (ANOVA) followed by Bonferroni post hoc multi-comparison test was utilized to analyze the statistical results. Statistical significances were set when $p<0.05$.

\section{Results}

\subsection{Scoparone ameliorates OGD/R-induced neuron injury}

To investigate the effect of scoparone on hippocampal neurons, cells were treated with different doses of scoparone $(0,25,50$, $100,150$ and $200 \mu \mathrm{M})$ for $48 \mathrm{~h}$. Data presented in Fig. 1B indicated that treatment with 150 and $200 \mu \mathrm{M}$ scoparone had significant effect on cell viability, however, cell viability of hippocampal neurons was not changed by scoparone at concentrations of 25,50 and $100 \mu \mathrm{M}$. Therefore, $25-100 \mu \mathrm{M}$ of scoparone was used in the following experiments. Furthermore, 
A

\section{B}
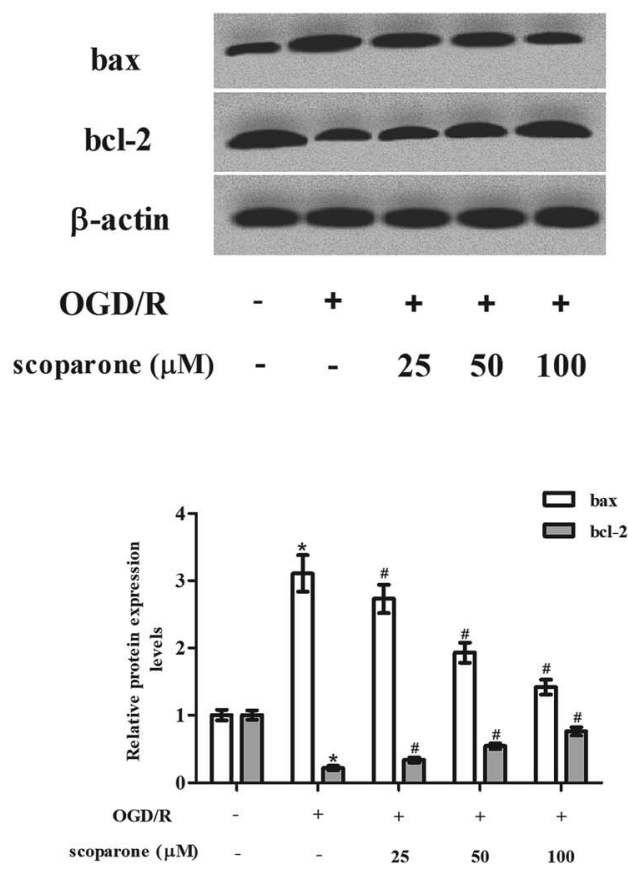

Fig. 3 Effect of different treatments on cell apoptosis in hippocampal neurons. (A) Cell apoptosis in hippocampal neurons with different treatments were measured using cell death detection ELISA kit. (B) Expression levels of bcl-2 and bax in hippocampal neurons with different treatments were detected using western blot. $n=3$. "*" indicates significant difference in comparison to the control group, $p<$ 0.05. "\#" indicates significant difference in comparison to the OGD/R induction group, $p<0.05$.

we observed that there was a significant decrease in cell viability of hippocampal neurons after $\mathrm{OGD} / \mathrm{R}$ induction. However, pretreatment with scoparone improved the cell viability of OGD/ R-induced hippocampal neurons (Fig. 1C).

\subsection{Scoparone represses $\mathrm{OGD} / \mathrm{R}$-induced oxidative stress in hippocampal neurons}

In order to evaluate the effect of scoparone on oxidative stress in hippocampal neurons, the oxidative-related markers including ROS generation, MDA level and activities of SOD and GPx were measured. The data in Fig. 2A and B indicated that marked increase in ROS generation and MDA level were observed in the
OGD/R-induced hippocampal neurons. Scoparone pretreatment attenuated the induction of ROS generation and MDA level. Besides, the activities of SOD and GPx were significantly decreased after OGD/R induction. Scoparone pretreatment caused significant elevation of SOD and GPx activities in OGD/ R-induced hippocampal neurons (Fig. 2C and D).

\subsection{Scoparone suppresses OGD/R-induced cell apoptosis in hippocampal neurons}

Then, we examined the effect of scoparone on cell apoptosis. Data presented in Fig. 3A represented that OGD/R induced cell apoptosis in hippocampal neurons, while scoparone suppressed the induction. In addition, the expressions of bax and bcl-2 were measured using western blot. The OGD/R-induced hippocampal neurons exhibited increased expression level of bax, as well as decreased expression level of bcl-2 as compared to the control cells. The changes in expressions of bax and bcl-2 caused by $\mathrm{OGD} / \mathrm{R}$ induction were mitigated in the scoparone pretreated cells (Fig. 3B).

\subsection{Scoparone induces the activation of Nrf2/HO-1 pathway in hippocampal neurons induced by $\mathbf{O G D} / \mathrm{R}$}

Next, the effect of scoparone on the Nrf2/HO-1 pathway was examined by detecting the expression levels of Nrf2 and HO-1. Results of western blot showed that OGD/R caused slight induction of Nrf2 and HO-1 expressions, while scoparone treatment enhanced OGD/R-induced increase in Nrf2 and HO-1 expressions (Fig. 4A). Furthermore, to validate the role of Nrf2/ HO-1 pathway in the protective effects of scoparone, the pathway was blocked by knockdown of Nrf2. Hippocampal neurons were transfected with si-Nrf2 to knockdown Nrf2. As shown in Fig. 4B, the expression levels of Nrf2 and HO-1 were markedly reduced after si-Nrf2 transfection. Moreover, si-Nrf2 transfection abolished the protective effect of scoparone on cell viability (Fig. 4C). The decreased level of ROS and increased activity of SOD caused by scoparone were reversed after si-Nrf2 transfection (Fig. 4D-E). Besides, the inhibitory effect of scoparone on cell apoptosis was mitigated by si-Nrf2 transfection (Fig. $4 \mathrm{~F}$ ).

\section{Discussion}

Ischemic stroke is the second leading cause of deaths, accounting for $9 \%$ of deaths worldwide. ${ }^{4}$ Cerebral I/R injury has been found to be important for the pathogenesis of ischemic stroke. ${ }^{2}$ ROS, the main type of free radicals released when reperfusion happens, play vital roles in the progress of cerebral $\mathrm{I} / \mathrm{R}$ injury. ${ }^{8,16}$ Accumulation of excessive ROS results in oxidative stress and overwhelms antioxidant defences, which leads to oxidative damage. Besides, ROS production triggers a chain of inflammatory responses including activation of inflammatory cells, as well as release and interaction of inflammatory mediators including cytokines, chemokines, and cell adhesion molecules, which can contribute to the cell injury. ${ }^{16}$ In addition, ROS disturbs various signal transductions such as MAPK, PI3K/ Akt, JAK-STAT, and NF-кB signaling pathways, thus leading to 
A

B

C

$\mathbf{E}$
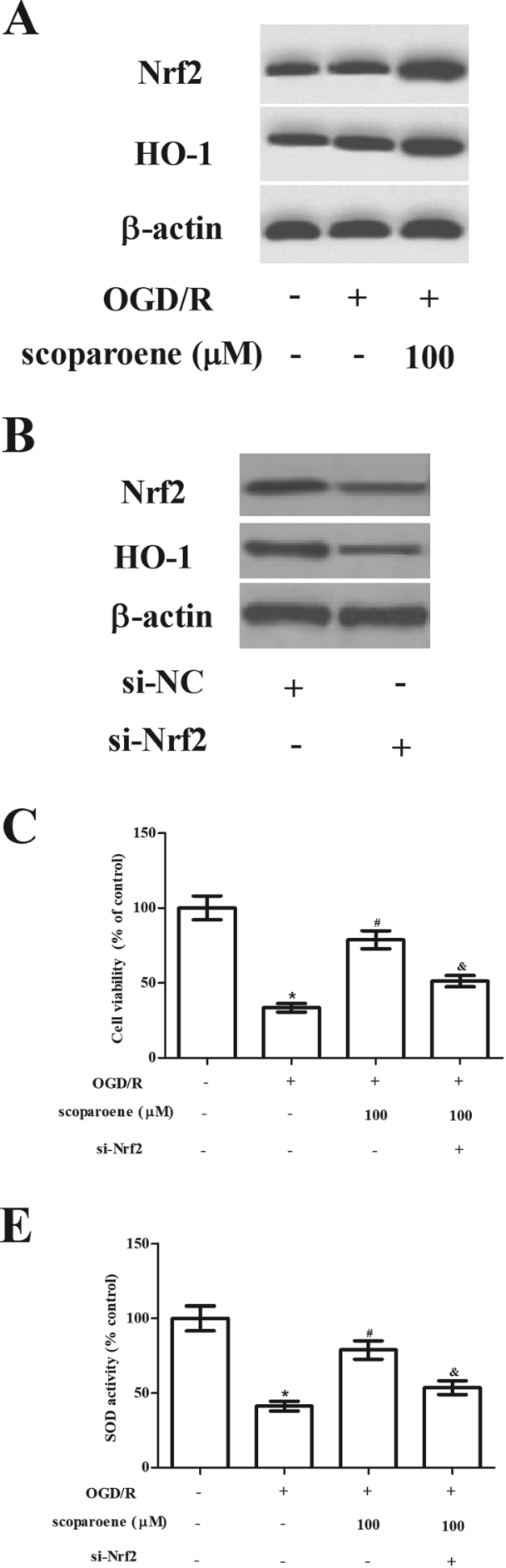
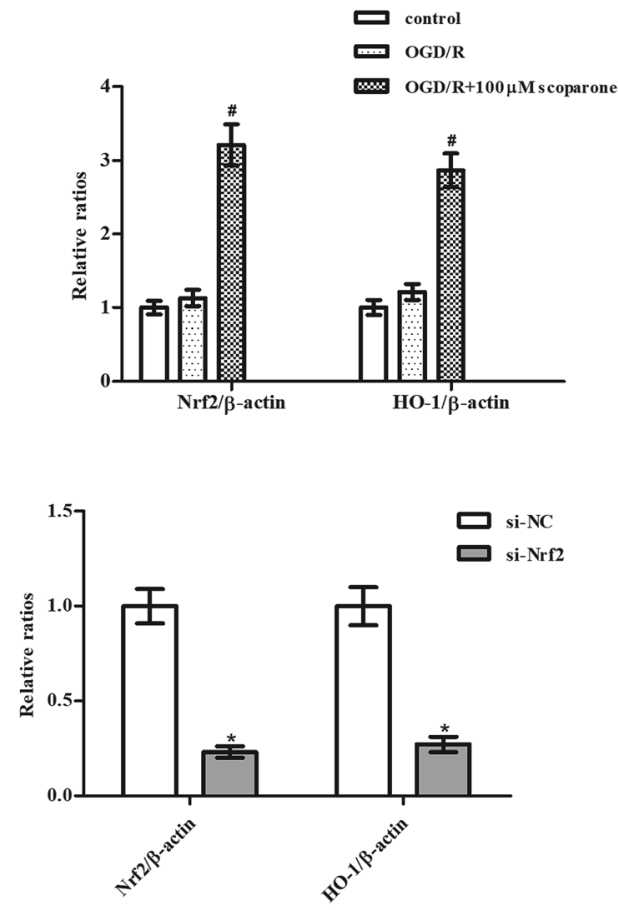

D

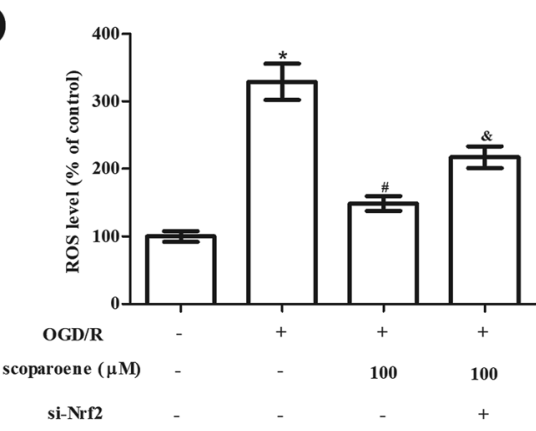

F

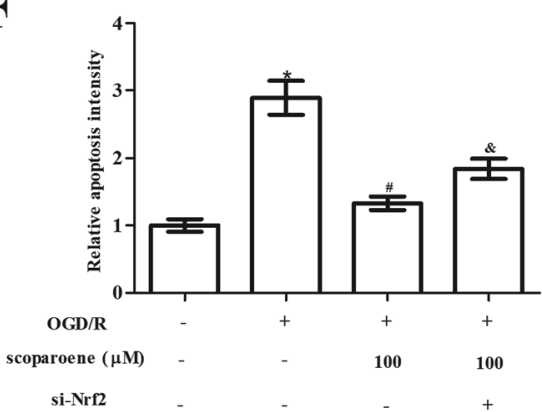

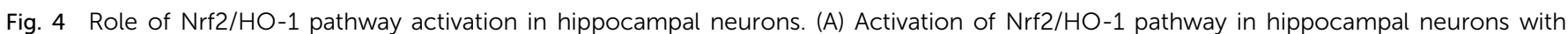

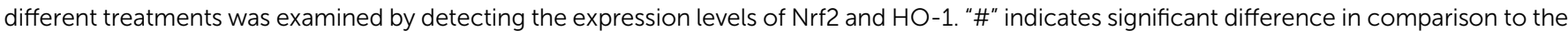

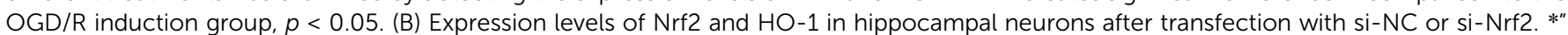

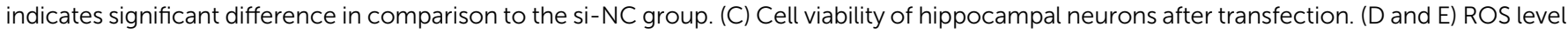

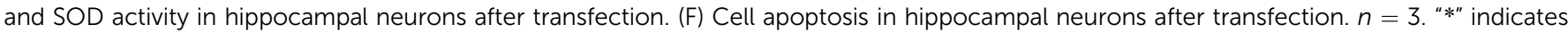

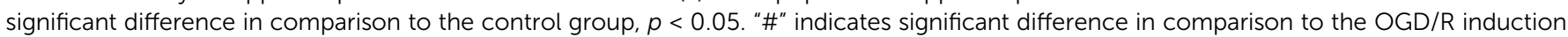
group, $p<0.05$. " $\&$ " indicates significant difference in comparison to the OGD/R+ scoparone (100 $\mu M)$ group, $p<0.05$.

cell apoptosis. ${ }^{2}$ Collectively, inhibition of ROS generation and oxidative stress might be effective and feasible strategies for reduction or minimization of cerebral reperfusion injury.
Scoparone is a natural compound that has been verified to possess anti-oxidative effect. Lee et al. ${ }^{17}$ reported that scoparone dose-dependently attenuates the production of general ROS and 
superoxide anions. In addition, scoparone diminishes the expression of nicotinamide adenine dinucleotide phosphate (NADPH) oxidase 1 (Nox1), which catalyzes the production of superoxide free radicals. ${ }^{16}$ Furthermore, scoparone elevates the expressions of SOD1 and catalase (CAT), which are responsible for the decomposition of free radicals. ${ }^{16}$ These findings suggest scoparone exerts strong anti-oxidative activity. Scoparone has been found to exhibit beneficial effect on neurological disorder. Scoparone attenuates pilocarpine (Pilo)-induced seizures in mice, as evidenced by the suppression of blood brain barrier breakdown, and inflammation and apoptosis in hippocampus and cortex. ${ }^{18}$ In vitro assays prove that scoparone represses LPSinduced inflammation and apoptosis in astrocytes. ${ }^{18}$ Moreover, scoparone exhibits protective effect against I/R-induced myocardial injury in an in vivo rat model. Besides, scoparone markedly improved cell viability of OGD/R-induced primary cardiac myocytes. ${ }^{13}$ Scoparone inhibits lactate dehydrogenase (LDH) release, MDA production and ROS production, and expressions of bax, caspase- 3 and cytochrome c, while induces expressions of SOD and bcl-2 in OGD/R-induced primary cardiac myocytes. ${ }^{13}$ In the present study, we found that scoparone improved cell viability of OGD/R-induced hippocampal neurons. Scoparone protected hippocampal neurons against OGD/R-induced oxidative stress and apoptosis.

Nrf2 is a master transcription factor that participates in the phase II antioxidant response, which is considered as the most important defense pathway to regulate oxidative stress derived damage. ${ }^{19,20}$ Under normal conditions, the Nrf2 binds to the repressor protein Kelch-like ECH-associated protein-1 (Keap1) and keeps in the cytoplasm at low levels. ${ }^{19}$ Under cellular stress, Nrf2 is released from Keap1 and trans locates to nucleus. Then Nrf2 binds to antioxidant response elements (ARE) and regulates the induction of transcriptional induction of multiple detoxifying enzymes such as HO-1.19,20 The Nrf2 signaling pathway has been established as the major mechanism of cellular defense against oxidative stress in a wide array of diseases including cerebral I-R injury. ${ }^{21} \beta$-Caryophyllene (BCP) pretreatment ameliorates oxidative damage and neuronal apoptosis in rats model of cerebral I-R injury, which might be mediated by upregulating the expression of Nrf2 and HO-1.22 Luo et al. ${ }^{23}$ proved that total flavonoids of Abelmoschus esculentus L. flowers exhibits protective effect on transient cerebral I/R injury through activation of the Nrf2-ARE pathway. The demonstration of the great importance of Nrf2 pathway in protecting the brain against I-R injury suggests that Nrf2 is a promising therapeutic target for the treatment of stroke. In the present study, we evaluated whether scoparone could affect the Nrf2 signaling pathway. The results showed that scoparone enhanced the activation of $\mathrm{Nrf} 2 / \mathrm{HO}-1$ signaling pathway in OGD/R-induced hippocampal neurons. Furthermore, knockdown of Nrf2 abolished the protective effect of scoparone on OGD/R-induced hippocampal neurons, implying that the protective effects of scoparone were mediated by $\mathrm{Nrf} 2 / \mathrm{HO}-1$ signaling pathway.

There existed several limitations in the present study. Firstly, we only evaluated the effect of scoparone on OGD/R-induced hippocampal neurons in vitro. An in vivo animal study will be considered in the following studies. Secondly, we did not add the treatment with nimodipine as a positive control, which may better reveal the protective effect of scoparone on cerebral I/R injury.

\section{Conclusion}

Taken together, our study demonstrated that scoparone protected hippocampal neurons from OGD/R-induced injury as proved by the improved cell viability, decreased oxidative and cell apoptosis. The protective effects of scoparone on OGD/Rinduced hippocampal neurons were partially attributed to the activation of Nrf2/HO-1 signaling pathway. Our knowledge will provide a theoretical foundation for further studying the effects of scoparone on cerebral I/R injury in vivo and a new mechanistic insight into the neuro-protective effects of scoparone.

\section{Conflicts of interest}

No conflict of interests.

\section{Abbreviation}

$\mathrm{I} / \mathrm{R}$

OGD/R

ROS

MDA

SOD

GPx

Nrf2

HO-1

DMSO

RIPA

ECL

CAT

$\mathrm{LDH}$

ARE
Ischemia/reperfusion

Oxygen-glucose deprivation/reperfusion

Reactive oxygen species

Malondialdehyde

Superoxide dismutase

Glutathione peroxidase

Nuclear factor E2-related factor 2

Heme oxygenase-1

Dimethyl sulfoxide

Radioimmunoprecipitation assay

Enhanced chemiluminescence

Catalase

Lactate dehydrogenase

Antioxidant response elements

\section{References}

1 G. A. Donnan, M. Fisher, M. Macleod and S. M. Davis, Lancet, 2008, 371, 1612-1623.

2 J. Yang, M. Chen, R. Y. Cao, Q. Li and F. Zhu, Adv. Exp. Med. Biol., 2018, 1087, 309-325.

3 S. A. Randolph, Workplace Health \& Safety, 2016, 64, 444.

4 R. Rodrigo, R. Fernandez-Gajardo, R. Gutierrez, J. M. Matamala, R. Carrasco, A. Miranda-Merchak and W. Feuerhake, CNS Neurol. Disord.: Drug Targets, 2013, 12, 698-714.

5 K. R. Turley, L. H. Toledo-Pereyra and R. U. Kothari, J. Invest. Surg., 2005, 18, 207-218.

6 H. K. Eltzschig and T. Eckle, Nat. Med., 2011, 17, 1391-1401.

7 J. H. Heo, S. W. Han and S. K. Lee, Free Radical Biol. Med., 2005, 39, 51-70.

8 P. H. Chan, J. Cereb. Blood Flow Metab., 2001, 21, 2-14. 
9 J. K. Kim, J. Y. Kim, H. J. Kim, K. G. Park, R. A. Harris, W. J. Cho, J. T. Lee and I. K. Lee, PLoS One, 2013, 8, e80391. 10 C. Lu, Y. Li, S. Hu, Y. Cai, Z. Yang and K. Peng, Biomed. Pharmacother., 2018, 106, 1169-1174.

11 A. Witaicenis, L. N. Seito, A. da Silveira Chagas, L. D. de Almeida, Jr., A. C. Luchini, P. Rodrigues-Orsi, S. H. Cestari and L. C. Di Stasi, Phytomedicine, 2014, 21, 240-246.

12 Y. J. Yang, H. J. Lee, H. S. Huang, B. K. Lee, H. S. Choi, S. C. Lim, C. K. Lee and M. K. Lee, J. Neurosci. Res., 2009, 87, 1929-1937.

13 C. Wan, Y. Wei, J. Ma and X. Geng, Mol. Med. Rep., 2018, 18, 1752-1760.

14 D. Y. Cho, H. M. Ko, J. Kim, B. W. Kim, Y. S. Yun, J. I. Park, P. Ganesan, J. T. Lee and D. K. Choi, Molecules, 2016, 21, 1718-1727.

15 A. J. Krohn, E. Preis and J. H. Prehn, J. Neurosci., 1998, 18, 8186-8197.
16 J. Pan, A. A. Konstas, B. Bateman, G. A. Ortolano and J. PileSpellman, Neuroradiology, 2007, 49, 93-102.

17 S. H. Lee and H. D. Jang, Exp. Cell Res., 2015, 331, 267-277. 18 J. Xia, C. Y. Li, H. Wang, Q. M. Zhang and Z. M. Han, Biomed. Pharmacother., 2018, 97, 1501-1513.

19 T. W. Kensler, N. Wakabayashi and S. Biswal, Annu. Rev. Pharmacol. Toxicol., 2007, 47, 89-116.

20 T. Nguyen, P. Nioi and C. B. Pickett, J. Biol. Chem., 2009, 284, 13291-13295.

21 R. Zhang, M. Xu, Y. Wang, F. Xie, G. Zhang and X. Qin, Mol. Neurobiol., 2017, 54, 6006-6017.

22 J. Lou, G. Cao, R. Li, J. Liu, Z. Dong and L. Xu, Neurochem. Res., 2016, 41, 1291-1304.

23 Y. Luo, H. X. Cui, A. Jia, S. S. Jia and K. Yuan, Oxid. Med. Cell. Longevity, 2018, 2018, 8987173. 